\title{
A micro fluorescent activated cell sorter for astrobiology applications
}

\author{
Donald W. Platt* ${ }^{\mathrm{a}}$, Richard B. Hoover ${ }^{\mathrm{b}}$ \\ ${ }^{a}$ Micro Aerospace Solutions, Inc., 2280 Pineapple Ave, Melbourne, FL 32935; \\ 'NASA/NSSTC, SD-50, 320 Sparkman Dr., Huntsville, AL 35805, USA
}

\begin{abstract}
A micro-scale Fluorescent Activated Cell Sorter ( $\mu$ FACS) for astrobiology applications is under development. This device is designed to have a footprint of $7 \mathrm{~cm} \times 7 \mathrm{~cm} \times 4 \mathrm{~cm}$ and allow live-dead counts and sorting of cells that have fluorescent characteristics from staining. The $\mu \mathrm{FACS}$ system takes advantage of microfludics to create a cell sorter that can fit in the palm of the hand. A micron-scale channel allows cells to pass by a blue diode which causes emission of marker-expressed cells which are detected by a filtered photodetector. A small microcontroller then counts cells and operates high speed valves to select which chamber the cell is collected in (a collection chamber or a waste chamber). Cells with the expressed characteristic will be collected in the collection chamber. This system has been built and is currently being tested. We are also designing a system with integrated MEMS-based pumps and valves for a small and compact unit to fly on small satellite-based biology experiments.
\end{abstract}

Keywords: Microfluidics, cell sorting, cell counting, live-dead staining, fluorescence

\section{INTRODUCTION}

A novel miniature instrument to assess in-situ cell viability as a function of exposure to microgravity and the space environment is under development The instrument is a microfluidics-based fluorescent activated cell sorter ( $\mu$ FACS) that is small enough to be flown aboard a free-flying CubeSat or micro-satellite to carry out viability studies of frozen anaerobic and aerobic bacterial cells.

This device is designed to have a footprint of $7 \mathrm{~cm} \times 7 \mathrm{~cm} \times 4 \mathrm{~cm}$ and to allow live-dead bacterial counts and sorting of

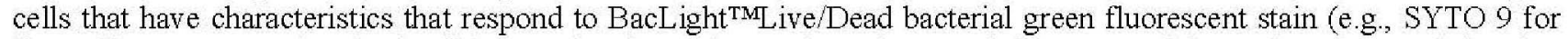
live cells or SYTOX for dead cells) or molecular probes such as the BacLight ${ }^{\mathrm{TM}}$ Bacterial Membrane Potential probe. Since these molecular probes use nucleic acid stains, they can distinguish live or dead biological cells from abiotic particles. The $\mu \mathrm{FACS}$ system takes advantage of microfluidics to create a miniaturized cell sorter that can fit in the palm of the hand. This staining system has been previously demonstrated with extermophilic bacteria. ${ }^{1}$

The detection of living Pleistocene bacteria in the Fox Tunnel and Antarctic Ice ${ }^{2}$ suggests that ice encased bacteria might survive for long periods in microgravity and the space environment. This enhances the feasibility of the transfer of microorganisms by impact ejection phenomena. If cross-contamination of Solar System bodies might have resulted from natural processes, perhaps some of the Planetary Protection Protocols that must be implemented for future spacecraft could be relaxed at additional cost savings to NASA. This device can be used to determine the viability of this hypothesis in a small nanosatellite or CubeSat system by carrying samples of bacteria in ice exposed to space.

The test $\mu$ FACS System could also be used to determine if living or dead bacteria are present in the upper crust (2-5 meters) of Europa. This would be much cheaper than developing a cryobot to penetrate the $3-4 \mathrm{~km}$ thick ice to reach the liquid water oceans.

*dplatt@micro-a.net; phone 1321 243-4633; fax 1928 396-3256; micro-a.net 


\section{SYSTEM DESIGN}

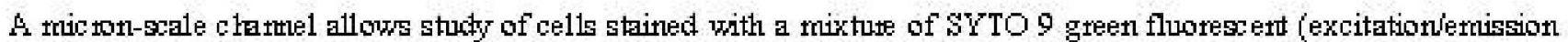
maxima of $480,500 \mathrm{tmn}$ ) and the ted propidium iodide (excitation/entiscion traxitna of 4901635 ) nucleic acid staits. The

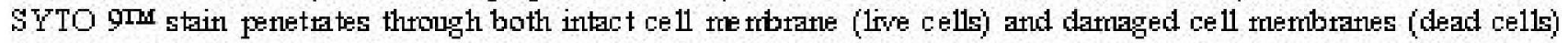

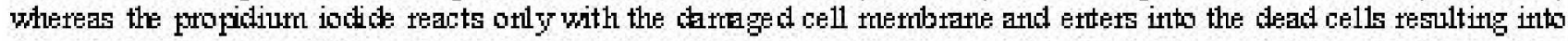
ted fluoregent cells.

Sirce it does not react with the ittact metrbrate, live cells fluoresce green. The stained cells enter the rrictofluidics capillaries and pass by the blue light encitting cliode (LED) which causes the stained live cells to fluotege gteetl and the deac cells to fluorese ted. The fluorescence emitted by cells is detected by a filtered photodetector. A strall trictocontroller the of opetates high speed valves to divett the cell to the collection chariber (live cells) or the waste chariber (dead cells). We take advantage of frior experience with micro-scale valve techrology to cteate a system with integrated MEMS-based pumpr and valves for a crnall and compact unit to fly on strall satellite based biology expetiments. Figure 1 shows a schematic of cutrent system and Figute 2 shows the integtated prototype electronics anc1 mictofludidics assetribly.

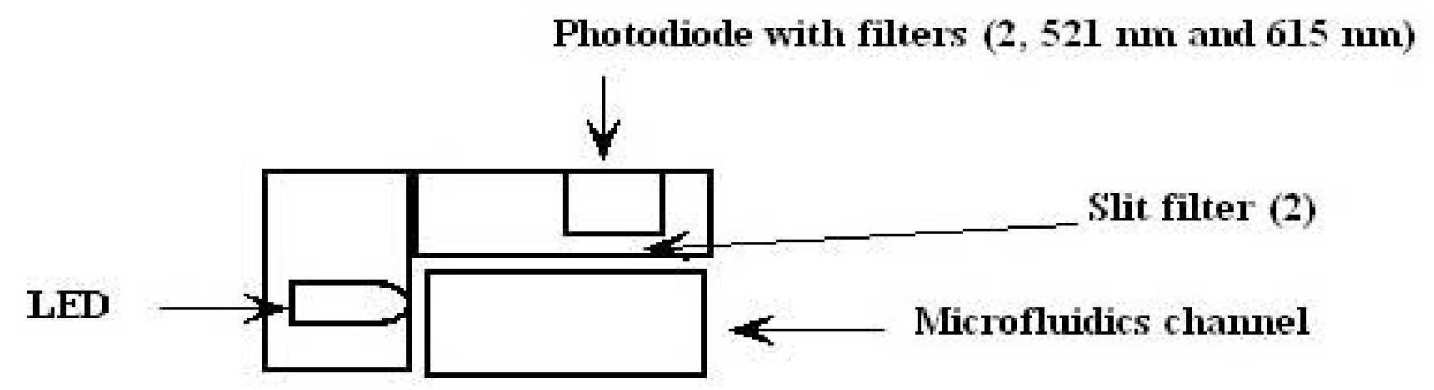

(Side)

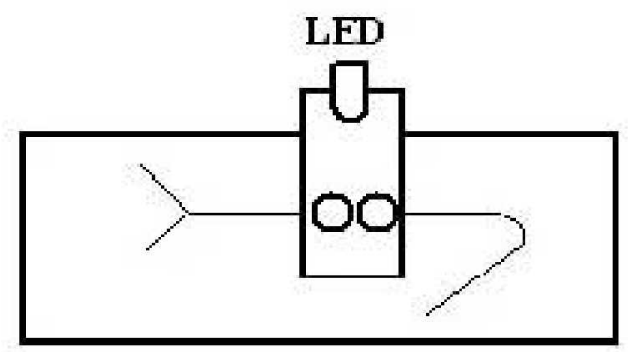

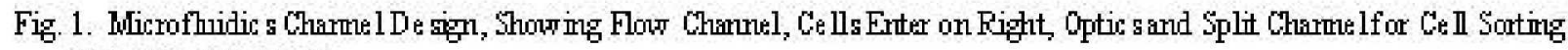
(I weilDead Sortimg)

A basis microfluidic chantel and electrotics control system has been developed and is undergoitg testimg. The text phase of this project will take the tricrofluidics chantuel and integtate valwing and controls to allow cells to be automatic ally fed into the system and then sent to one of the toro chambets (one for live celle and one for dead cells as incicated by staining).

Fhotodiodes with atyropuate wavelength filte ts observe the flow chatthel and associated electronicr count the celle and

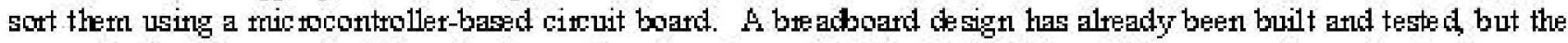
current system is manually fed and only counts cells and provides live-dead ratice. We ate currently evaluating how we

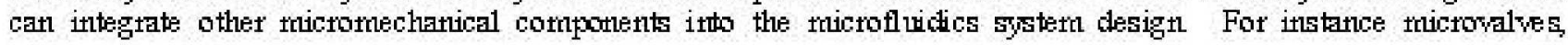


microlenses and micropumps could be incorporated into the $\mu$ FACS system design to make the system more compact and efficient. A variety of microoptics systems are also under evaluation.
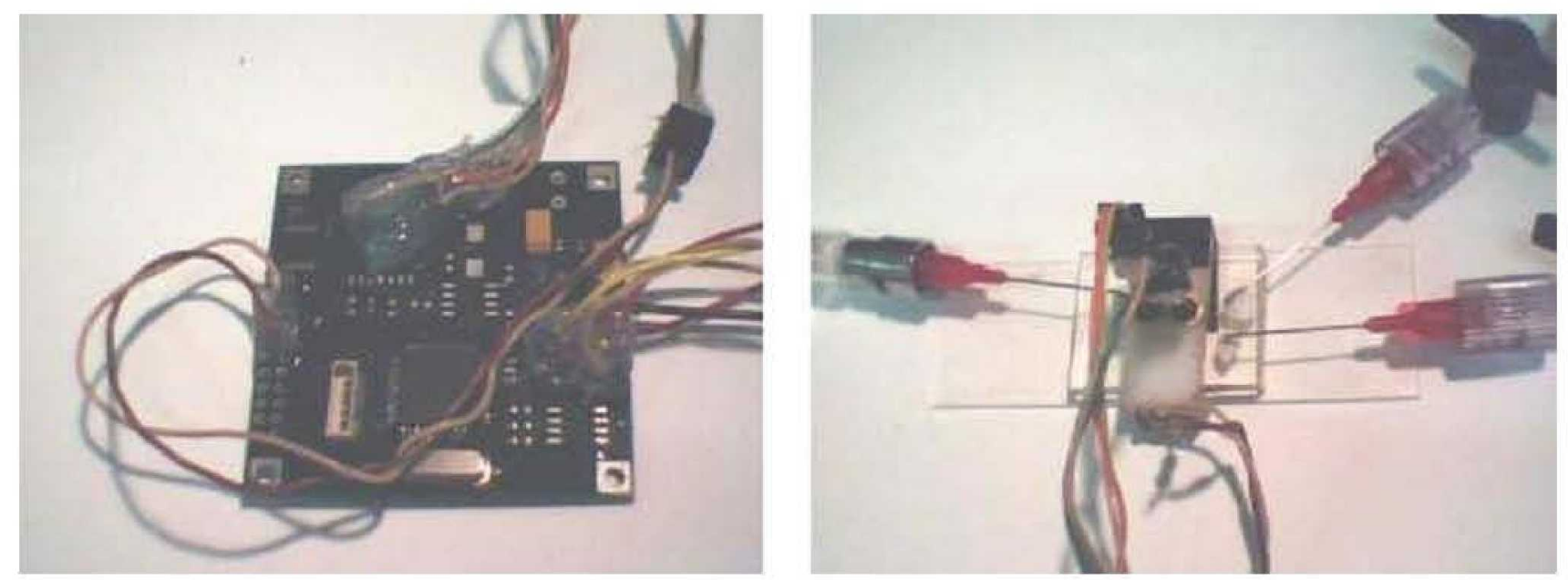

Fig. 2. Protype System (Electronics, left and Mi crofluidics, right).

The end goal will be to create a complete astrobiology lab-on-a-chip system to sample bacteria, count the live-dead ratio over time and then sort the bacteria for further analysis as seen in Figure 3. This system integrates microfluidics, optics, control electronics and valves in a package that can easily fit into a $3 \mathrm{U}$ CubeSat with multiple systems to study different types of bacteria and/or different bacteria exposures. The system can also be adaptable to different excitation LEDs and filter wavelengths for detection.

Overall system mass is 100 grams within the footprint of $7 \mathrm{~cm} \mathrm{x} 7 \mathrm{~cm} \mathrm{x} 4 \mathrm{~cm}$. It uses under $100 \mathrm{~mA}$ of power to process a bacterial sample. The LED has emission centered at $485 \mathrm{~nm}$. The optics system consists of micro lenses with filters optimized for green and red emission for SYTO 9 and red propidium iodide, respectively. A photodiode measures emission intensity. Simple green/red ratios can be measured using the two ch annels to determine perc ent live bacteria or more sophisticated analysis can take place and bacteria samples separated based upon viability. Figure 3 shows the final system design. 


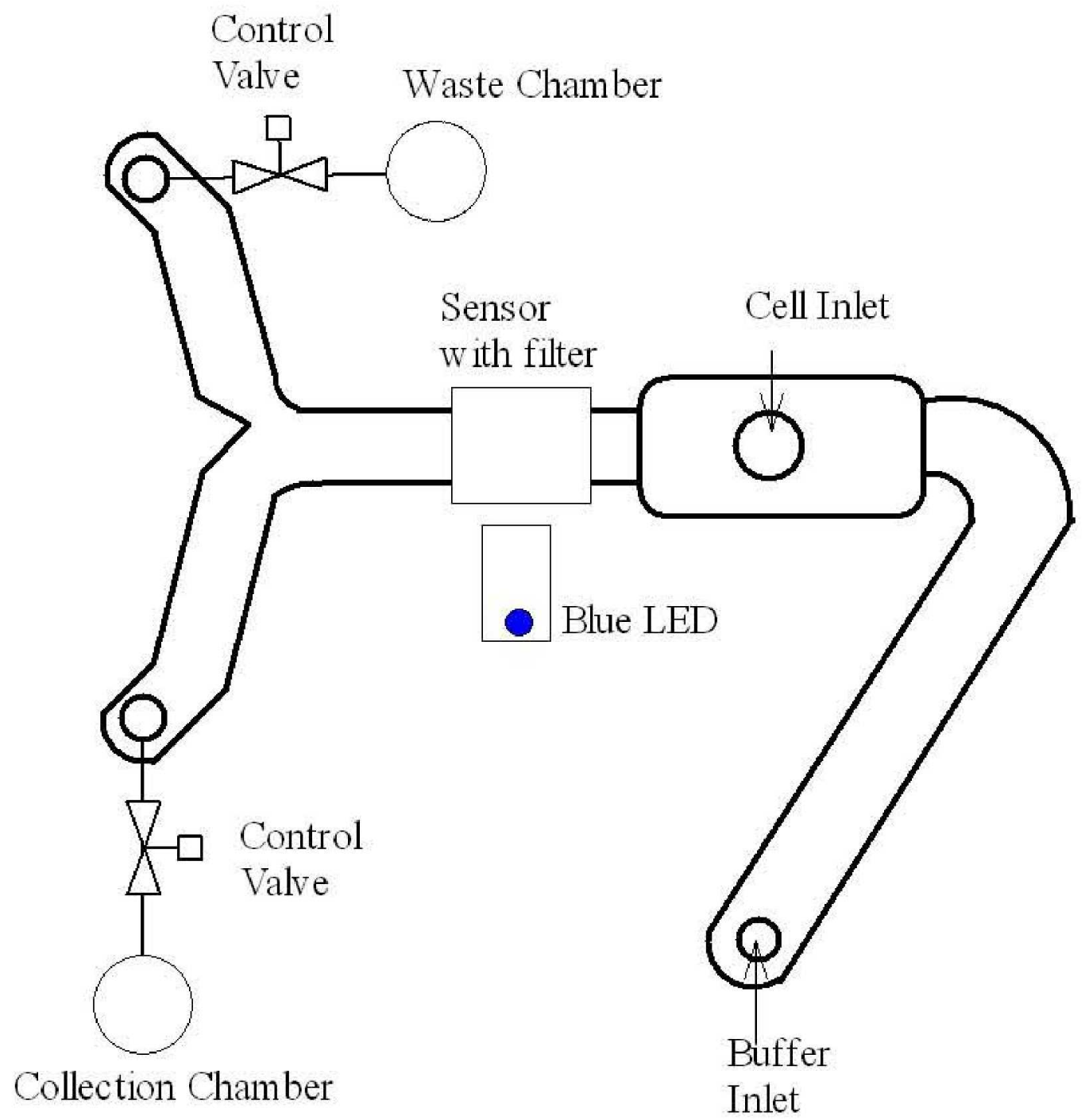

Fig. 3 System Design Schenatic Show the Microfluides Flow Channel with Sheathing Buffer Inlet and Cell Inlet, Blue Illumination LED (for emission) and red/gree filtered photodetector output. A microcontroller counts cells and controls valves for collection.

A sheathing flow is used to hydrodynamically focus the cells. Wolff $e t$ al. implemented a "smoking chimney" focusing technique that is used in the system described in this paper. ${ }^{3}$ An important factor will be the required relative flow rate of cell-suspended fluid compared to the buffer fluid flow. Increasing the ratio of sheath flow to sample flow increases the focusing of the system. The transverse injection of the cell-suspension fluid should provide the proper level of mixing even in a microgravity environment. With microcontroller-based control of sheathing flow and sample flow, the system can be optimized for various conditions and focusing can be done "on the fly". This focusing flow is illustrated in Figure 4.

Other focusing techniques require microfabrication of electrodes on both the bottom and top of the channel, precision alignment of these electrodes, el ectronics for generating electric AC field, and conductivity of the focusing liquid. These additional complications are eliminated in this design allowinga smaller, more compact design ideal for small satellite systems. 


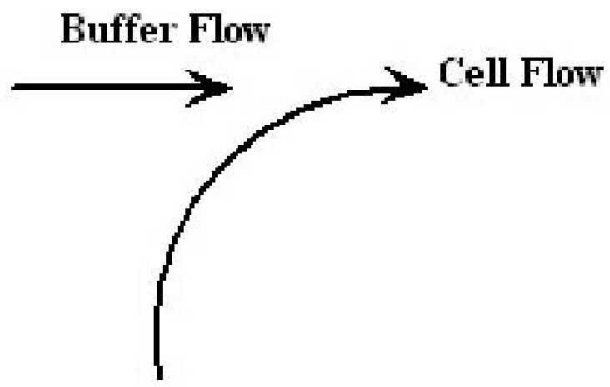

Side View

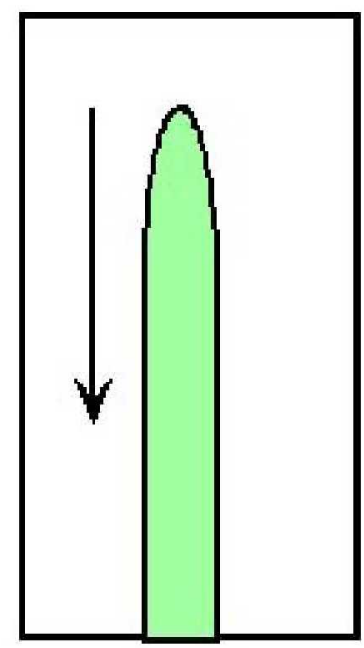

\section{Top View}

Fig. 4 Microchannel sheathing (buffer) flow and focusing of the introduced bacterial fluid. Arrows indicate flow direction.

Figure 5 shows BacLight ${ }^{\mathrm{TM}}$ Live/Dead stain of Spirochaeta americana pure culture from Owens Lake, CA showing live cells (green) and dead cells (red). This illustrates the ease in distinguishing between viable and dead bacteria with this staining technique. New high intensity, yet miniature LEDs with emissions around 470 to $490 \mathrm{~nm}$ allows this technology to be implemented in a microfluidics system.

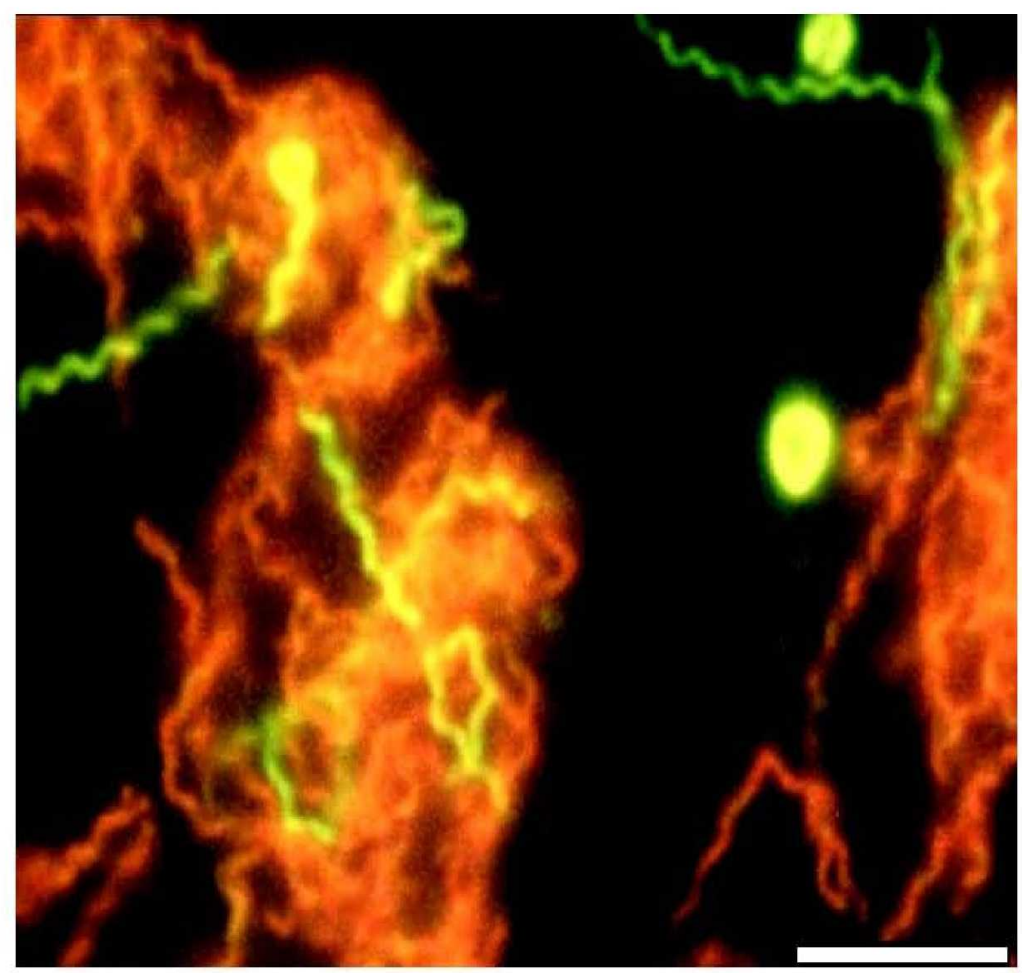

Fig. 5 BacLight ${ }^{\mathrm{TM}}$ Live/Dead stain of Spirochaeta americana pure culture from Owens Lake, CA showing live cells (green) and dead cells (red). 


\section{APPLICATIONS}

This system relies on technology based on Live/Dead Molecular probes and microfluidics capillary to permit in-situ bioanalytical assessment of the viability of selected anaerobic bacteria and archaea thawed out after different periods of exposure to the space environment. This work has drwan on expertise in microfluidics-based system combined with recent discoveries of survivability of microbial extremophiles entrapped in the ancient ice from the Fox Tunnel of Alaska, Deep Vostok Ice Cores as well as more modern ice from the Anuchin Glacier, Ice Sculptures and Ice-Bubbles studied in-situ during the 2008 Antarctic Expeditions.

This instrumentation can have valuable commercial applications and permit significant cost reductions to future Space Missions. A miniature, lightweight, low-power instrument that can distinguish live bacteria from dead bacteria and bacterial cells in ice from abiotic particles could be incorporated into future SMD Planetary Science Missions. Verification that microbial life entrained in ice can remain viable suggests that trans-planetary transfer of intact microbiota may have occurred during great cosmic impacts.

Recently there was a discovery of living Pleistocene microorganisms, (Carnobacterium pleistocenium, sp. nov.) that had been frozen in the ice of the Fox Tunnel of Alaska for 32,000 years and was still alive and expressed a motility immediately as the ice thawed. This finding implies that ancient living bacteria may be crypopreserved in frozen water ice glaciers at the Polar Caps of Mars. It also means that it may not be necessary to drill through several kilometers of ice to penetrate the crust of icy moons such as Europa or Enceladus to search for life. Living microbes may be present within the first few centimeters or meters of the ice crust, if they are protected from the UV and radiation environment by the ice sheet. These icy moons have become of great interest to Astrobiology due to indications of liquid water oceans beneath their icy crusts. However, viable microorganisms have been discovered in the permafrost and frozen thermokarst ponds of Siberia and Alaska (Carnobacterium pleistocenium). During Antarctic Expeditions, living and physiologically active microorganisms were observed in abundance in freshly thawed ice from the Anuchin glacier and ice bubbles of Lake Untersee and the frozen ice sheets covering the Schirmacher Oasis lakes (unpublished data). These facts expand the possibility of surviving of microbial extremophiles on the Polar Ice Caps of Mars, and the crusts of other frozen bodies of our Solar Systems. Understanding the nature and distribution of microbial life in the glaciers and perennially ice covered lakes of Antarctica should provide meaningful and valuable data required to develop and evaluate instruments and mission operational techniques to search for and recognize evidence of extant or extinct life during future Mars Missions or during explorations of other Solar System bodies. This compact device can be used to analyze bacteria in-situ in these environments. Combining this mFACS with a compact imaging instrument would create a complete field laboratory that is transportable in a backpack.

The possibility that viable microorganisms entrapped in ice may survive exposure to microgravity and the space environment enhances the feasibility of transfer of microorganisms via impact ejection phenomena. If ice-entrapped microorganisms remain viable in the space environment and can be revived on melting then it is feasible to search for living bacteria in the uppermost 2-5 meters of the crust of Europa or Enceladus using the $\mu F A C S$ system. This is much less expensive than developing robotic systems to penetrate a $3-4 \mathrm{~km}$ thick ice shell to reach liquid water. Ice is an excellent absorber of high energy particles - only $20 \mathrm{~cm}$ of ice reduces the flux of $100 \mathrm{MeV}$ protons to $1 / \mathrm{e}$. Demonstrating the feasibility of cryo-panspermia could have implications to the NASA Origins Program concerning distribution and Evolution of Life in the Cosmos and the Origin of Life on Earth as well as the NASA Planetary Protection Program. If cross-contamination of Solar System bodies can feasibly occur by natural impact ejection transfer of ice containing viable microbes, it may be possible to relax Planetary Protection Protocols at significant cost savings to NASA.

Potential commercial applications of the device include lab-on-a-chip systems, field-based medical instruments and astronaut health monitoring systems to measure blood counts or pathogen activity on long duration space missions. Since the system can be tailored to counting other cell-types and particles; these devices may be used for counterbioterrorism efforts by detecting potentially deadly microbiological agents in their intact states. Very small, portable medical research devices may be used for pathogen diagnostics in field settings in the rural US and developing countries. Their low cost, ease of use, small footprint and low power consumption could bring state of the art medical care to areas of the world which have not previously had such services. A main issue previously has been the large size, complex support equipment, and cost associated with many FACS systems. 


\section{FUTURE WORK}

The newy developing field of CubeSat-based science missions is the perfect setting to test the $\mu F A C S$ system and the wiability of bacteria submerged in ice in the space environment. NASA has previously flown G enesat and Pharmasat. 3U CubeSats with total spacecraft masses under 5 kilograms. ${ }^{4}$ This system can be flown on a similar CubeSat platform for astrobiology research into bacterial wiability in space. A simple $3 \mathrm{U}$ CubeSat concept is shown in Figure 6.

An exposure section can be configured on the bottom and top surfaces of the spacectaft (the circle in Figure 6 ). This exposure area would then interface to a valve manifold system to allow bacterial samples to be made at desired initervals during the mission. For instance, each week or month a sample could be taken and bacterial wiability analyzed using the $\mu F A C S$ dewice.

A series of ground tests will also be conducted with watious extremophile bacteria to gain a system baseline and for calibration purposes. The system can also be tested with bacteria in extreme Eath environments such as the polar regions and radiation chambers.

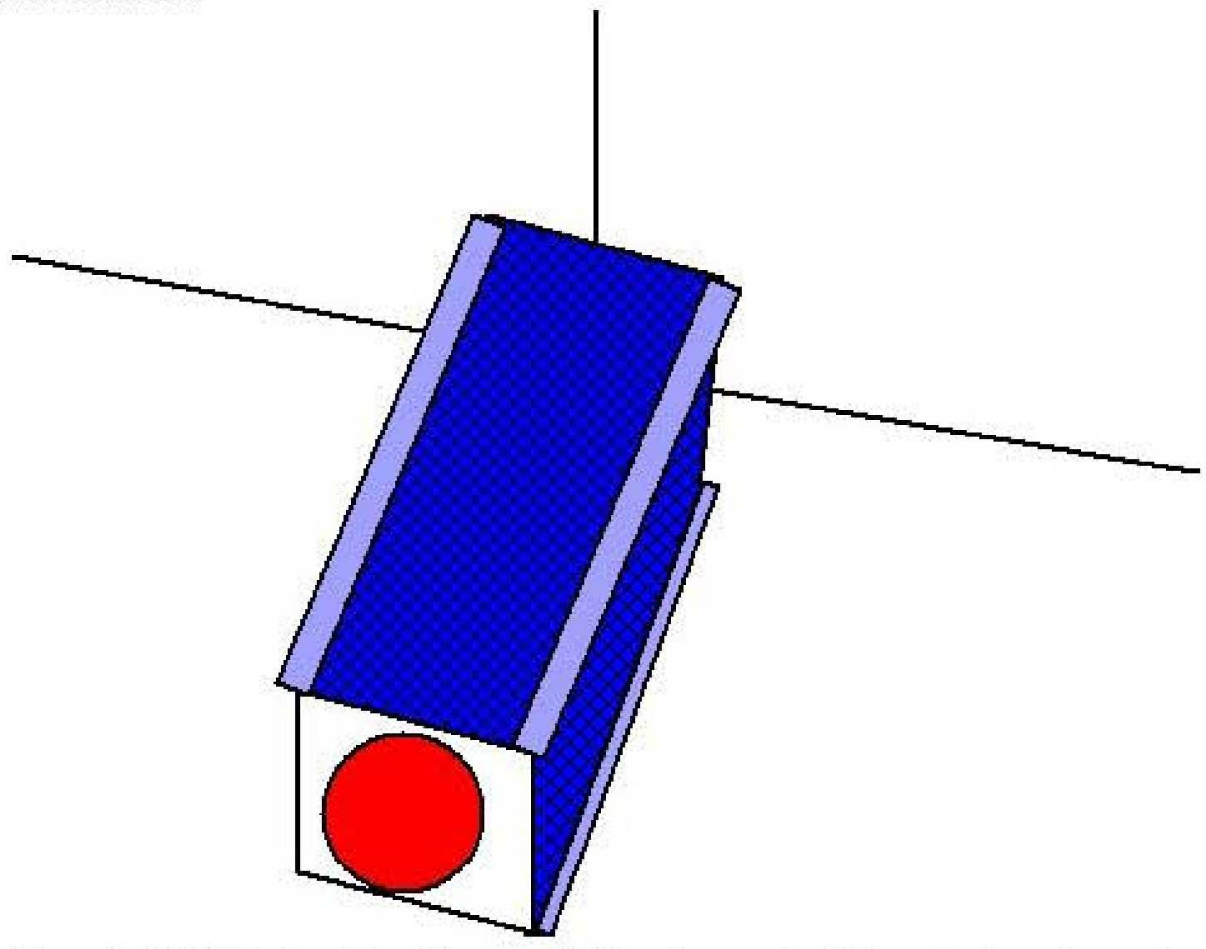

Figure 6 A $3 \mathrm{U}$ CubeSat with anEx posure Platform (bottom circle) for anice-bacteria sample

\section{CONCLUSION}

The mFA CS system offers a low cost and responsive solution for astrobiology missions to analyze bacterial wiability in space as well as detect life on other solar system bodies and analyze bacteria in remote locations on Earth. The design is based upon the fundamental principles that a microfluidic- $\mu \mathrm{FACS}$ system in a CubeSat format that would function in conjunction with a well established commercially awailable fluorescent dye-based microbial detection technology that allows specific identification of the liwe cells from dead. This overall system makes a low-cost, low-mass and lowpower astrobiology lab-on-a-chip. 
We are working towards the development of a flight instrument prototype that could form the basis for future flight instruments to address the most fundamental question of Astrobiology: Is life restricted to Planet Earth or is it distributed throughout the Cosmos?

\section{REFERENCES}

[1] Leuko, S., Legat, A., Fendrihan, S., and Stan-Lotter, H., "Evaluation of the LIVE/DEAD BacLight Kit for Detection of Extremophilic Archaea and Visualization of Microorganisms in Environmental Hypersaline Samples", AEM, 70 (11), 6884-6886(2004).

[2] Pikuta, E., Marsic, D., Bej, A., Tang, J., Krader, P., and Hoover, R. B., "Carnobacterium pleistocenium sp. nov., a novel psychrotolerant, facultative anaerobe isolated from permafrost of the Fox Tunnel in Alaska", /IJS, 55, 473-478 (2005).

[3] Wolff, A., Perch-Nielsen, I. R., Larsen, U.D., Friis, P., Goranovic, G., Poulsen, C. R., Kutter, J. P., and Telleman, P., "Integrating advanced functionality in a microfabricated high-throughput fluorescent-activated cell sorter", Lab Chip, 3 , $22-27$ (2003).

[4]Parra, M, McGinnis, M.R., Ricco, A.J., Yost, B., and Hines, J.W., "Studying space effects on microorganisms autonomously: genesat, pharmasat and the future of bio-nanosatellites", Gravitational and Space Biology 21(2), 9-17 (June 2008). 